\title{
Improving distance estimates to nearby bright stars: Combining astrometric data from Hipparcos, Nano-JASMINE and Gaia
}

\author{
Daniel Michalik, ${ }^{1}$ Lennart Lindegren, ${ }^{1}$ David Hobbs, ${ }^{1}$ \\ Uwe Lammers, ${ }^{2}$ and Yoshiyuki Yamada ${ }^{3}$ \\ ${ }^{1}$ Lund Observatory, Lund University, Box 43, SE-22100 Lund, Sweden \\ e-mail: [daniel.michalik, lennart, david] Castro.lu.se \\ ${ }^{2}$ European Space Agency (ESA/ESAC), P. O. Box 78, ES-28691 Villanueva de la Cañada, \\ Madrid, Spain \\ e-mail: uwe.lammers@sciops.esa.int \\ ${ }^{3}$ Department of Physics, Kyoto University, Oiwake-cho Kita-Shirakaw Sakyo-ku, Kyoto, \\ 606-8502 Japan \\ e-mail: yamada@amesh.org
}

\begin{abstract}
Starting in 2013, Gaia will deliver highly accurate astrometric data, which eventually will supersede most other stellar catalogues in accuracy and completeness. It is, however, limited to observations from magnitude 6 to 20 and will therefore not include the brightest stars. NanoJASMINE, an ultrasmall Japanese astrometry satellite, will observe these bright stars, but with much lower accuracy. Hence, the Hipparcos catalogue from 1997 will likely remain the main source of accurate distances to bright nearby stars. We are investigating how this might be improved by optimally combining data from all three missions through a joint astrometric solution. This would take advantage of the unique features of each mission: the historic brightstar measurements of Hipparcos, the updated bright-star observations of Nano-JASMINE, and the very accurate reference frame of Gaia. The long temporal baseline between the missions provides additional benefits for the determination of proper motions and binary detection, which indirectly improve the parallax determination further. We present a quantitative analysis of the expected gains based on simulated data for all three missions.
\end{abstract}

Keywords. astrometry, catalogs, methods: data analysis, methods: statistical, reference systems

\section{Introduction}

The distance to a star can most directly be deduced from its trigonometric parallax. From the ground, this was only done for a few thousand very nearby stars, but with the advent of space astrometry this picture changed dramatically. Hipparcos (1989-1993) was the first satellite to determine astrometric parameters (stellar positions, parallaxes and proper motions) from space and yielded the distances to approximately 21,000 stars with an uncertainty of better than 10\% (ESA 1997). Now, 25 years later, Gaia will further improve our knowledge of stellar astrometry significantly and provide millions of stellar parallaxes with unprecedented accuracy. Gaia is an ESA cornerstone mission which will be launched for its nominal five-year mission at the end of 2013. It will continuously scan the sky in a well-chosen pattern and observe up to a billion stars down to magnitude 20. However, because of CCD saturation it will not observe stars brighter than about magnitude 6 . Hence the brightest $\sim 5000$ stars are not observed by Gaia, and for these Hipparcos will continue to be a main source of distance information.

In addition to these two astrometry missions, the third upcoming mission is the ultrasmall Japanese satellite Nano-JASMINE. Just like Gaia, this satellite is based on CCD 


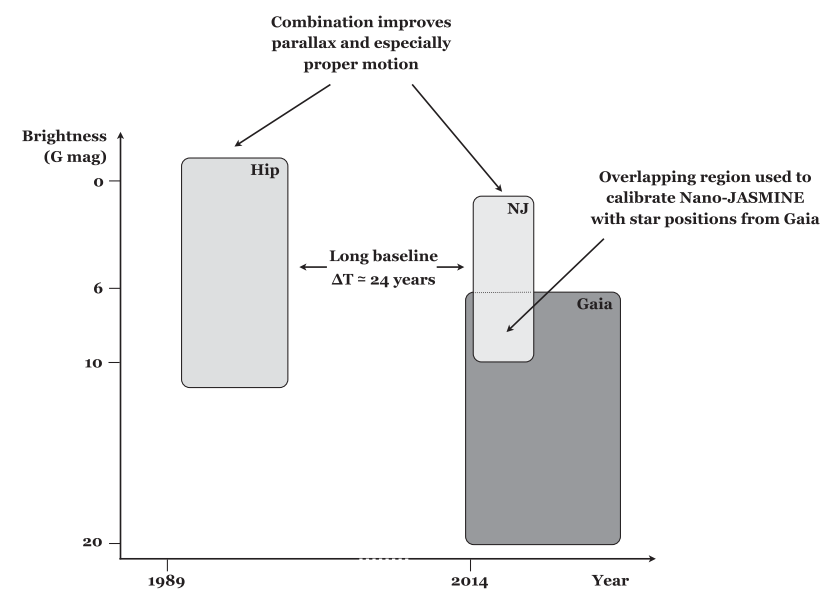

Figure 1. Magnitude range versus mission time for the three astrometry missions, Hipparcos ('Hip'), Nano-JASMINE ('NJ') and Gaia.

detections, and its scanning principle and observing strategy are derived from the Gaia pendants. This mission, however, is meant to be a technology demonstrator for larger follow-up missions and is therefore significantly smaller and less accurate. It is scheduled for launch by the end of 2013 and expected to provide astrometry for approximately one million stars in the visual magnitude range from $\sim 1$ to 10 with an accuracy of $\sim 3$ mas for objects of magnitude 7.5. Gaia data will be reduced using the Astrometric Global Iterative Solution (AGIS), developed by ESA and Lund Observatory (Lindegren et al. 2012). Thanks to a collaboration between the Nano-JASMINE Science Team and parts of the AGIS team, it is possible to use AGIS also for the core data reduction of Nano-JASMINE.

\section{Catalogue combination by joint solution}

While Nano-JASMINE's uncertainties are not better than the uncertainties in the Hipparcos results, significant improvements for bright-star astrometry can be made by combining the results of Hipparcos and Nano-JASMINE, thanks to the long time baseline between the missions. The combination is done by incorporating the Hipparcos information directly in the astrometric solution for the Nano-JASMINE data. This is done using the Hipparcos data and the inverse of its covariances as starting values when accumulating the normal equations for the astrometric solution. In contrast to a posteriori catalogue combination, this 'joint solution' combines the data sets in a statistically optimal way (Michalik et al. 2012), taking into account the correlations between the different astrometric parameters.

In addition to the astrometric improvement facilitated by the combination described above, further improvement can be gained by incorporating (preliminary) results from Gaia during the Nano-JASMINE data processing: Gaia and Nano-JASMINE will both observe stars between magnitude 6 and 10. Since the astrometry of these stars is welldetermined by Gaia, a joint solution can be used to determine the attitude and geometry deviations of Nano-JASMINE with better accuracy and therefore improve all NanoJASMINE results. This includes calibrating the basic angle (the nominally fixed angle between the two fields of view of the satellite) which may be affected by thermal variations originating in the low-earth-orbit of the satellite. The basic angle stability is particularly critical to avoid zero-point errors thus allowing the determination of absolute parallaxes. Additionally, the Nano-JASMINE results are aligned with the Gaia reference frame. 
Therefore, optimal results for bright stars are obtained by reducing the Nano-JASMINE data together with preliminary Gaia results and by combination of these with the historic Hipparcos measurements during data analysis (see Fig. 1). We quantify the expected improvements in parallax and proper-motion determination by simulating this scenario.

\section{Simulations}

Simulations are carried out using AGISLAB, a software package developed at Lund Observatory to aid the development of algorithms for Gaia data processing (Holl et al. 2012). It is used to simulate Nano-JASMINE observations and process these data in the same manner as done for the real mission, i.e., by employing the AGIS algorithms. AGIS is an iterative scheme that implements a block-wise least-squares solution on very large data sets, i.e., as required to reduce data from large astrometry missions such as Gaia. Nano-JASMINE simulations are based on a realistic scanning law featuring a triangular spin-axis precession motion (see Fig. 2). The observation accuracy model assumes a (somewhat optimistic) centroiding uncertainty of $1 / 300^{\text {th }}$ of a pixel $(\sim 7$ mas) for stars brighter than magnitude 7 , with additional photon noise for fainter stars ( 230 mas at magnitude 10).

The simulation data set consists of two parts. The first is composed of the 5026 brightest Hipparcos stars $(\mathrm{mag}<6)$, with astrometric parameters and uncertainties taken from the Hipparcos Catalogue. They are used to evaluate the improvement in the uncertainties of the astrometric parameters of the bright stars. To these, we add 330,000 randomly distributed stars of magnitude 10 that represent stars observed in the overlapping magnitude range which were not included in the Hipparcos Catalogue. They represent the stars that will be seen by Gaia as well as by Nano-JASMINE, and for which the well-determined Gaia positions help to better constrain the solution.

First, we simulate the errors in the Hipparcos Catalogue, providing a reference case for the bright stars and initial values for the solutions (Cases B and C below), which incorporate the Hipparcos data. This is followed by three simulations.

Case A: We simulate Nano-JASMINE observations with the two data sets mentioned above and process the data without additional information, i.e. Nano-JASMINE only.

Case B: In a second run, we incorporate the information from Hipparcos into the data processing of Nano-JASMINE using the method described in Michalik et al. (2012).
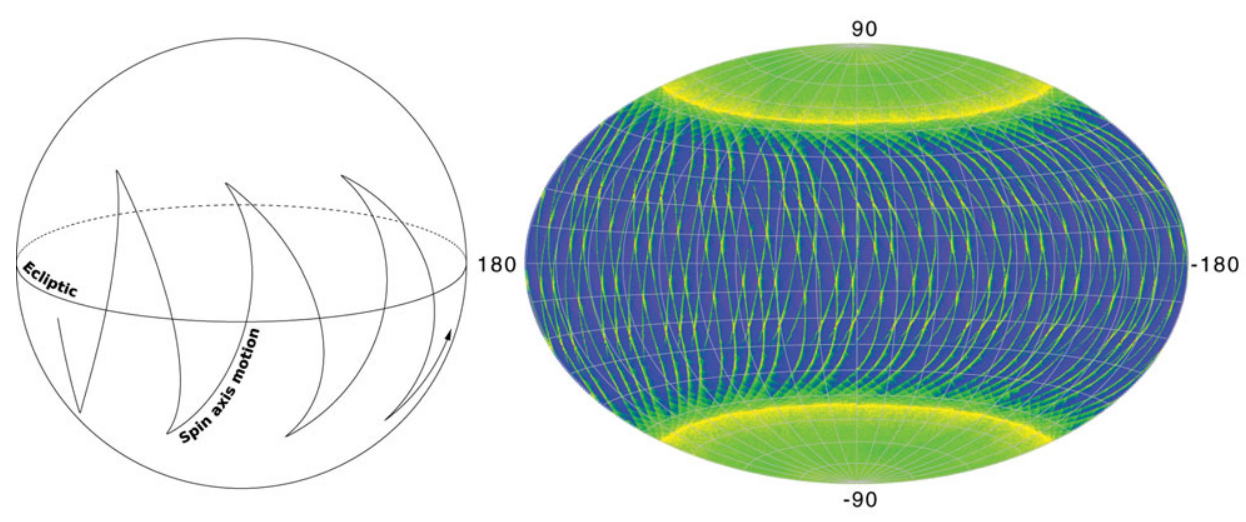

Figure 2. (left) 130 days of spin-axis motion. (right) Number of observations per star (ecliptical sky map), based on simulations of two years of the baseline Nano-JASMINE scanning law. 
Table 1. Average uncertainties (RSE $\dagger$ ) of 5026 Hipparcos stars between magnitude 1 and 6 .

\begin{tabular}{llcc}
\hline & & Parallax $(\mu \mathrm{as})$ & Proper motion $\left(\mu \mathrm{as} \mathrm{yr}^{-1}\right)$ \\
\hline Reference & Hipparcos only (Hip) & 740 & 673 \\
Case A & Nano-JASMINE only $($ NJ) & 1282 & 1844 \\
Case B & Hip + NJ & 595 & 50 \\
Case C & Hip + NJ + Gaia & $\mathbf{5 8 8}$ & $\mathbf{4 3}$ \\
\hline
\end{tabular}

Case C: In a third run, we simulate the astrometric parameters with Gaia accuracy and fold expected Gaia covariances (de Bruijne 2012) into the Nano-JASMINE data processing. This is done by setting up the covariances of these sources with a $\sigma$ according to the expected Gaia uncertainties and using it in a joint solution scheme. Under the optimistic assumption of uncorrelated parameters, the covariance is set up with zeros in all off-diagonal positions.

\section{Results}

We compare the three cases and our current knowledge of the bright-star astrometric uncertainties with the results in Table 1 . Our current knowledge is represented by the Hipparcos Catalogue. The results of the Nano-JASMINE observations alone are less precise, but combined with Hipparcos they lead to a significant improvement with respect to our current knowledge. Including provisional Gaia results yields a further improvement thanks to the improved calibration of the geometry and attitude of Nano-JASMINE. This improvement is fairly small, but it needs to be emphasized that there is a second and very important advantage of the Nano-JASMINE joint solution with Gaia results, i.e. alignment of the Nano-JASMINE results with the Gaia reference frame.

\section{Conclusions}

Nano-JASMINE offers an opportunity to significantly improve the Hipparcos parallaxes and proper motions of the brightest $\sim 5000$ stars which will not be observed by Gaia. In addition, a combined solution with Gaia data ensures that the results are in the same reference frame as the Gaia catalogue and that the parallaxes are absolute.

\section{References}

de Bruijne, J. H. J. 2012, ApESSS, 341, 31

ESA 1997, The Hipparcos and Tycho Catalogues, ESA SP-1200

Holl, B., Lindegren, L., \& Hobbs, D. 2012, A\& A, 543, A15

Lindegren, L., Lammers, U., Hobbs, D., O’Mullane, W., Bastian, U., \& Hernández, J. 2012, $A \& 3,538, \mathrm{~A} 78$

Michalik, D., Lindegren, L., Hobbs, D., Lammers, U., \& Yamada, Y. 2012, Astron. Soc. Pac. Conf. Ser., 461, 549

$\dagger$ "The Robust Scatter Estimate (RSE) is defined as 0.390152 times the difference between the $90^{\text {th }}$ and $10^{\text {th }}$ percentiles of the distribution of the variable. For a Gaussian distribution, it equals the standard deviation. The RSE is used as a standardized, robust measure of dispersion [within the Gaia core processing unit]." (Lindegren et al. 2012) 